\title{
Supporting Information for Highly Alkyl-Selective Addition to Ketones with Magnesium Ate Complexes Derived from Grignard Reagents
}

\author{
Manabu Hatano, Tokihiko Matsumura, Kazuaki Ishihara* \\ Graduate School of Engineering, Nagoya University, \\ Furo-cho, Chikusa, Nagoya 464-8603, Japan
}

General Methods. Infrared (IR) spectra were recorded on a JASCO FT/IR 460 plus spectrometer. ${ }^{1} \mathrm{H}$ NMR spectra were measured on a Varian Gemini-2000 spectrometer $(300 \mathrm{MHz})$ at ambient temperature. Data were recorded as follows: chemical shift in ppm from internal tetramethylsilane on the TM scale, multiplicity ( $\mathrm{s}=$ singlet; $\mathrm{d}=\mathrm{doublet}$; $\mathrm{t}=$ triplet; $\mathrm{m}=$ multiplet), coupling constant $(\mathrm{Hz})$, integration, and assignment. ${ }^{13} \mathrm{C} \mathrm{NMR}$ spectra were measured on Varian Gemini-2000 (75 MHz) spectrometer. Chemical shifts were recorded in ppm from the solvent resonance employed as the internal standard (deuterochloroform at $77.00 \mathrm{ppm}$ ). Low-resolution mass analysis (LRMS) and GC analysis were performed with a Shimadzu GC/MS instrument [GC-17A/QP-5050A; column: TC-1 $(0.25 \mathrm{~mm}$ x $30 \mathrm{~m})$ ] by direct insertion for chemical ionization $(\mathrm{CI})$ with isobutane.

All experiments were carried out under an atmosphere of dry nitrogen. For thin-layerchromatography (TLC) analysis throughout this work, Merck precoated TLC plates (silica gel 60GF254 $0.25 \mathrm{~mm}$ ) were used. The products were purified by column chromatography on silica gel (E. Merck Art. 9385). Visualization was accomplished by UV light (254 nm), anisaldehyde, $\mathrm{KMnO}_{4}$ and phosphomolybdic acid.

In experiments that required dry solvents, diethyl ether (dehydrate), toluene (dehydrate), dichloromethane (dehydrate), tetrahydrofuran (dehydrate) and hexane (dehydrate) were purchased from Kanto Chemical Co., Inc. MeLi (0.98 M in diethylether, Kanto Chemical Co., Inc.; 1.60 M in diethylether, Acros or Kanto Chemical Co., Inc.). $n$-BuLi (1.60 M in $n$-hexane, Kanto Chemical Co., Inc.). $\quad n$-BuMgCl (0.90 $M$ in THF, Kanto Chemical Co., Inc.). $t$-BuLi (1.48 $M$ in pentane, Kanto Chemical Co., Inc.). $\operatorname{EtMgBr}(0.96 M$ in THF, Kanto Chemical Co., Inc.). $i$-PrMgBr (0.65 $M$ in THF, Kanto Chemical Co., Inc.). $n$ - $\mathrm{PrMgBr}$ (1.04 $M$ in THF, Kanto Chemical Co., Inc.). $n-\mathrm{Bu}_{2} \mathrm{Mg}$ (1.00 $M$ in heptane, Aldrich). $\quad \mathrm{PhLi}$ (1.05 $M$ in cyclohexane-diethylether, Kanto Chemical Co., Inc.). $\mathrm{PhMgBr}$ (1.02 M in THF, Kanto Chemical Co., Inc.) 


\section{Representative procedure: For addition to acetophenone 5 by $n$-Bu $3 \mathrm{MgLi} / \mathrm{Bpy}$ reagents (Table 2 ).}

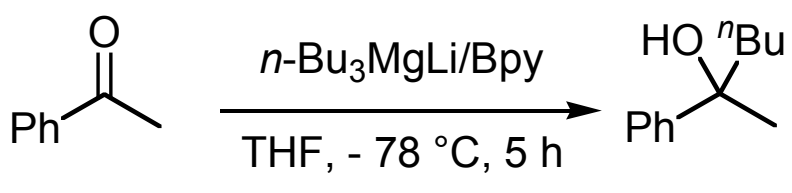

5

6

To a solution of 2,2'-bipyridyl in $1.5 \mathrm{~mL}$ of THF was added $n$ - $\mathrm{Bu}_{2} \mathrm{Mg}$ (1.06 mmol, $1.0 \mathrm{M}$ in $n$-heptane solution) and $n$-BuLi (1.0 mmol, $1.60 \mathrm{M}$ in $n$-hexane solution) at $-78{ }^{\circ} \mathrm{C}$ under nitrogen atmosphere. This suspention was stirred at that temperature for $1 \mathrm{~h}$. Then acetophenone $5(1.0 \mathrm{mmol})$ in $1.5 \mathrm{~mL}$ of THF was added. The mixture was stirred at $-78{ }^{\circ} \mathrm{C}$ for $5 \mathrm{~h}$, quenched by saturated aqueous $\mathrm{NH}_{4} \mathrm{Cl}(10 \mathrm{~mL})$, extracted with ether (10 $\mathrm{mL} \square 3)$ and washed by brine $(10 \mathrm{~mL})$. The combined organic layer was evaporated in vacuo and the residue was purified by short column chromatography (hexane/AcOEt $=5 / 1$ ) to afford 8 in $96 \%$ yield.

\section{Representative procedure: For ethylation to acetophenone 5 by $\mathrm{EtMe}_{2} \mathrm{MgLi}$ reagents} (Table 5).

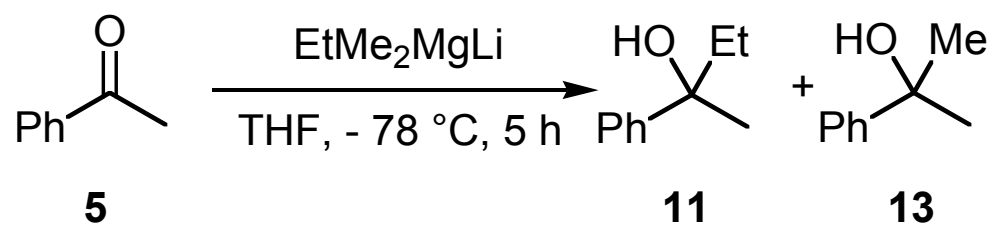

To $1.5 \mathrm{~mL}$ of THF was added MeLi (2.0 mmol, $1.60 \mathrm{M}$ in diethylether solution) and EtMgBr (1.2 mmol, $0.98 \mathrm{M}$ in THF solution) at $-78{ }^{\circ} \mathrm{C}$ under nitrogen atmosphere. This solution was stirred at that temperature for $1 \mathrm{~h}$. Then acetophenone $5(1.0 \mathrm{mmol})$ in 1.5 $\mathrm{mL}$ of THF was added. The mixture was stirred at $-78^{\circ} \mathrm{C}$ for $5 \mathrm{~h}$, quenched by saturated aqueous $\mathrm{NH}_{4} \mathrm{Cl}(10 \mathrm{~mL})$, extracted with ether $(10 \mathrm{~mL} \square 3)$ and washed by brine $(10 \mathrm{~mL})$. The combined organic layer was evaporated in vacuo and the residue was purified by short column chromatography (hexane/AcOEt $=5 / 1$ ) to afford the product 11 in $93 \%$ yield. 
2-phenyl-2-hexanol (2, Table 1) $)^{1,2,3}$

3-hydroxy-1,3-diphenyl-1-butanone $(3 \text {, Table } 1)^{4,5}$

3-phenyl-3-heptanol (6, Table 2, entry 2$)^{3}$

2-methyl-3-phenyl-3-heptanol (6, Table 2 , entry 3$)^{6}$

1,1-diphenylpentan-1-ol (6, Table 2 , entry 6$)^{3,7}$

1-butyl-1,2,3,4-tetrahydro-1-naphthol (6, Table 2, entry 7)

1,1-dicyclohexyl-1-pentanol (6, Table 2, entry 8$)^{9}$

1,1-diphenyl-1-pentanol (9, Table 3 , entry 1 ; Table 6 , entry 4$)^{3}$

triphenylmethanol (9, Table 3 , entry 2$)^{10,11}$

1,1-diphenylethanol (9, Table 3, entry 3; 10, Table 4 entries 2 and 4; 15, Table 6) $)^{10,12,13}$

1,1-diphenyl-1-propanol (9, Table 3 , entry 4; Table 6 , entry $1 ; \mathbf{1 1}$, Table 5 , entry 7$)^{10,14}$

2-phenyl-2-propanol (10, Table 4, entries 1 and 3; 13, Table 5, entry 1) $)^{1,15,16,17}$

3,3-dimethyl-2-phenyl-2-butanol (10, Table 4, entry 5; 13, Table 5, entry 4) $18,19,20,21$

2-phenyl-2-butanol (11, Table 5 , entry $1 ; \mathbf{1 3}$, Table 5 , entry 2$)^{1,2}$

3-phenyl-3-pentanol (11, Table 5, entry 2$)^{22}$

2-methyl-3-phenyl-3-pentanol (11, Table 5, entry 3$)^{23}$

2,2-dimethyl-3-phenyl-3-pentanol (11, Table 5, entry 4) ${ }^{24}$

2-(1-naphthyl)-2-butanol (11, Table 5, entry 5) ${ }^{25}$

2-(2-naphthyl)-2-butanol (11, Table 5, entry 6) ${ }^{26}$

1-ethyl-1,2,3,4-tetrahydro-1-naphthol (11, Table 5, entry 8$)^{27}$

2-(3-pyridinyl)-2-butanol (11, Table 5, entry 9) ${ }^{27}$

2-(4-pyridinyl)-2-butanol (11, Table 5, entry 10) ${ }^{27}$

1-(3-pyridinyl)ethanol (12, Table 5, entry 9) ${ }^{28}$

3-methyl-2-phenyl-2-butanol (13, Table 5, entry 3$)^{18}$

2-(1-naphthyl)-2-propanol (13, Table 5, entry 5) ${ }^{29}$

2-(2-naphthyl)-2-propanol (13, Table 5, entry 6$)^{30}$

1-methyl-1,2,3,4-tetrahydro-1-naphthol (13, Table 5, entry 8$)^{31,32,33}$

2-(3-pyridinyl)-2-propanol (13, Table 5, entry 9) ${ }^{34}$

2-(4-pyridinyl)-2-propanol (13, Table 5, entry 10) $)^{34}$

2-(1-naphthyl) -2-propanol (13, Table 5, entry 5) ${ }^{26}$

2-methyl-1,1-diphenyl-1-propanol (9, Table 6, entry 2$)^{10}$

1,1-diphenyl-1-butanol (9, Table 6 , entry 3$)^{35}$

$\mathbf{4}$ and $\mathbf{1 2}$ are commercially available from Aldrich. 
(1) Imamoto, T.; Kusumoto, T.; Tawarayama, Y.; Sugiura, Y.; Mita, T.; Hatanaka, Y.; Yokoyama, M. J. Org. Chem. 1984, 49, 3904.

(2) Imamoto, T.; Kusumoto, T.; Yokoyama, M. J. Chem. Soc., Chem. Commun. 1982, 1042.

(3) Inoue, H.; Suzuki, M.; Fujimoto, N. J. Org. Chem. 1979, 44, 1722.

(4) Wessjohann, L.; Wild, H. Synthesis 1997, 512.

(5) Allan, J. F.; Henderson, K. W.; Kennedy, A. R. Chem. Commun. 1999, 1325.

(6) Benkeser, R. A.; Siklosi, M. P.; Mozdzen, E. C. J. Am. Chem. Soc. 1978, 100, 2134.

(7) Yamanaka, H.; Miyano, N.; Hanafusa, T. J. Org. Chem. 1991, 56, 2573.

(8) Takeda, N.; Imamoto, T. Org. Synth. 1999, 76, 228.

(9) Einhorn, C.; Einhorn, J.; Luche, J.-L. Tetrahedron Lett. 1991, 32, 2771.

(10) Toda, F.; Takumi, H.; Yamaguchi, H. Chem. Express 1989, 4, 507.

(11) Pearce, P. J.; Richards, D. H.; Scilly, N. F. J. Chem. Soc., Perkin Trans. 1 1972, 1655.

(12) Guijarro, D.; Mancheňo, B.; Yus, M. Tetrahedron, 1993, 49, 1327.

(13) Ashby, E. C; Wiesemann, T. L.; Bowers, Jr., J. S.; Laemmle, J. T. Tetrahedron Lett. 1976, 17, 21

(14) Uchiyama, M.; Furumoto, S.; Saito, M.; Kondo, Y.; Sakamoto, T. J. Am. Chem. Soc. 1997, 119, 11425.

(15) Liang, X.; Bols, M. J. Chem. Soc., Perkin Trans. 1 2002, 503.

(16) Bartoli, G.; Bosco, M.; Dalpozzo, R. Tetrahedron Lett. 1985, 26, 115.

(17) Weidmann, B.; Seebach, D. Helv. Chimica. Acta. 1980, 63, 2451.

(18) Krief, A.; Clarembeau, M.; Barbeaux. P. J. Chem. Soc., Chem. Commun. 1986, 457.

(19) Bianchi, M.; Matteoli, U.; Frediani, P.; Menchi, G.; Piacenti, F.; Botteghi, C.; Marchetti, M. J. Organomet. Chem. 1983, 252, 317.

(20) Bassetti, M.; Floris B.; Illuminati, G. J. Organomet. Chem. 1980, 202, 351.

(21) Kratt, G.; Beckhaus, H.-D.; Lindner, H. J. Chem. Ber. 1983, 116, 3235.

(22) Kataoka, Y.; Akiyama H.; Makihara, I.; Tani, K. J. Org. Chem. 1997, 62, 8109.

(23) Guijarro, D.; Mancheňo, B.; Yus, M. Tetrahedron, 1994, 50, 8551.

(24) Duismann, W.; Ruchardt, C. Chem. Ber. 1973, 106, 1083.

(25) Acree, Jr, F.; Haller, H. L. J. Am. Chem. Soc. 1943, 65, 1652.

(26) Yus, M.; Ramoón, D. J.; Prieto, O. Tetrahedron: Asymmetry 2003, 14, 1103.

(27) Weber, B.; Seebach, D. Tetrahedron 1994, 50, 6117. 
(28) Bellezza, F.; Cipiciani, A.; Cruciani, G.; Fringuelli, F. J. Chem. Soc., Perkin Trans. 1 2000, 4439.

(29) Terao, Y.; Wakui, H.; Nomoto, M.; Satoh, T.; Miura, M.; Nomura, M. J. Org. Chem. 2003, 68, 5236.

(30) Markó, I. E.; Leung, C. W. J. Am. Chem. Soc. 1994, 116, 371.

(31) Noji, M.; Ohno, T.; Fuji, K.; Futaba, N.; Tajima, H.; Ishii, K. J. Org. Chem. 2003, 68, 9340.

(32) Rangarajan, R.; Eisenbraun, E. J. J. Org. Chem. 1985, 50, 2435.

(33) Adam, G.; Andrieux, J.; Plat, M. Tetrahedron 1982, 38, 2403.

(34) Epsztajn, J.; Bieniek, A. J. Chem. Soc., Perkin Trans. 1 1985, 213.

(35) Maercker, A.; Passlack, M. Chem. Ber. 1983, 116, 710. 